\title{
Categorización de los objetos que forman parte del Extended Self, y sus discursos asociados*
}

\section{Categorization of objects that are part of the extended self, and its associated discourses}

Recepción: 25 Febrero 2016 | Aprobación: 13 Julio 2017

\author{
Lucía Poggio \\ Universidad Complutense de Madrid, España \\ ORCID: http://orcid.org/0000-0002-7413-5560 \\ Juan I. Aragonés \\ Universidad Complutense de Madrid, España
}

a Autora de correspondencia. Correo electrónico: luciapoggio@psi.ucm.es

Para citar este artículo: Poggio, L., \& Aragonés, J. (2018). Categorización de los objetos que forman parte del Extended Self, y sus discursos asociados. Universitas Psychologica, 17(2), 1-10. https://doi. org/10.11144/Javeriana.upsy.17-2.coes

\section{RESUMEN}

La personalización del espacio se construye con las claves que el propietario distribuye en el espacio. Estas claves, mayoritariamente objetos, forman parte del Extended Self. Para comprobar si existe un discurso diferenciado según la categoría a la que pertenecen los objetos de los dormitorios, se realizaron tres estudios. Tras obtener una lista de los objetos más representativos, en el siguiente estudio los participantes clasificaron bajo su criterio estos objetos. Un análisis de cluster mostró dos categorías: funcional y simbólico. Finalmente, un análisis de contenido de las razones de selección de los objetos mostró discursos diferenciados para cada categoría. Los resultados confirman que los objetos se organizan en funcional y simbólico, en donde se obtienen discursos diferenciados según la categoría a la que pertenecen.

Palabras clave

personalización; extended self; funcional; simbólico; dormitorios.

\begin{abstract}
Personalization of space is constructed through the cues that the owner leaves in the space. These cues, mostly objects, are also a basic part of the extended self. To check if there is a differentiate discourse consistent with the categories in which the objects belong, three studies are carried out. After obtaining a list of the most representative, in the subsequent study the participants short these objects using their own criteria. A Cluster analysis showed two categories, Functional and Symbolic. Finally, a content analysis about the reasons of participants to choose each object showed differentiated discourses for each category. These results confirm the objects are organized into Functional and Symbolic, producing differentiated discourses. depending on the category they belong.

Keywords

personalization; extended self; functional; symbolic; bedrooms.
\end{abstract}

A medida que se produce un uso más cotidiano del espacio, las personas comienzan a apropiarse de este, modificándolo 
y adecuándolo a sus gustos y preferencias para alcanzar un mayor bienestar, llegando a convertirlo, en palabras de Cooper (1995), en "un espejo del self" sobre el que se proyectan los valores y creencias del residente. Es entonces cuando se puede comenzar a hablar de la personalización del espacio, entendiendo este concepto como el conjunto de huellas que el propietario deja en un lugar y que están vinculadas con valores, estatus, autodeterminación y preferencias (Becker, 1977).

Varios han sido los autores que, a través de la personalización de estos lugares, han pretendido observar qué rasgos del propietario es posible inferir por medio de la observación de sus viviendas. Entre ellos, Gosling, Ko, Mannarelli y Morris (2002) propusieron un modelo a través del cual los observadores hacen inferencias de determinados rasgos de los ocupantes basándose en las claves que estos disponen en sus lugares. Con las primeras evidencias de que dichas claves parecen proporcionar información relevante de los propietarios de ciertos lugares, Gosling, Craik, Martin, y Pryor (2005) desarrollaron el personal living space cue inventory (PLSCI), un cuestionario que comprende una parte de descriptores globales del lugar (e.g.quiet-noisy), y otra de contenidos específicos en la que se evaluaban 385 claves específicas, que abarcaban desde las paredes y techos hasta el método de sujeción de los posters. Así pretendían comprobar si los observadores serían capaces de inferir ciertas características del propietario a través de los indicadores o claves, depositados en los dormitorios por los mismos. Los resultados mostraron que estos indicadores proporcionaban información acerca del residente, concretamente el género y el rasgo de apertura a la experiencia.

Similar a estas investigaciones, es la realizada por Aragonés, Amérigo y Pérez-López (2010). Con el objetivo de estudiar los objetos que, presentes en los dormitorios, facilitan a los observadores inferir las características de personalidad del residente, los autores solicitaron a los participantes que, por medio de la observación de fotografías de dormitorios, indicasen qué señales les permitían identificar rasgos de la personalidad del propietario, así como variables sociodemográficas del mismo. A través de un análisis de correspondencias, los resultados mostraron que existe una relación entre los objetos funcionales, identificados por los observadores, y los rasgos de personalidad de competencias y habilidades (responsabilidad, estabilidad emocional y apertura a la experiencia), mientras que los elementos simbólicos se asociaban más a dimensiones sociales (extraversión y amabilidad).

Tal y como se ha observado en cada una de las investigaciones anteriormente comentadas, los espacios primarios tales como hogares, dormitorios y oficinas proporcionan información acerca de características del habitante por medio de la observación de dichos lugares y de las claves que se encuentran presentes en los mismos. La mayoría de las claves, de las que los observadores se valen para realizar sus inferencias, están basadas en los objetos y la distribución que su dueño ha hecho de ellos, lo que indica que estos pueden ser una rica fuente de información acerca de sus propietarios y del sello personal que ellos buscan reflejar (Gosling et al., 2005; Páramo, 2008).

Así mismo, diferentes investigaciones avalan que las personas realizan inferencias a diario acerca de lo que les rodea (Dittmar, 1992; Hall \& Andrzejeski, 2008). Por tanto, la mera observación de una persona interactuando con alguno de sus objetos personales (bien sea su maletín o un libro) da lugar a una inferencia de las características personales de esta. Más allá de que los objetos formen parte de la vida diaria, estos son más que meras posesiones materiales (Norman, 2004), ya que permiten al propietario elaborar un concepto de "quién soy" (Belk, 1992), convirtiéndolos en un medio para la personalización y auto-representación (Páramo, 2008). Así, para poder entender el significado de los objetos es necesario comprender que, consciente o inconscientemente, estos son vistos por su propietario como parte de su "yo" (Solomon, 2008).

El concepto de "yo extendido" es un término que acuña por primera vez Belk (1988), basándose, en parte, en el "yo empírico" de las personas, que James (1890) define como 
"todo lo que puede ser llamado como mío"(p. 233). Así, este término incluiría el cuerpo, las facultades psíquicas, la ropa, la vivienda, el cónyuge e hijos, amigos, creaciones, y un largo etcétera. De forma similar, Sartre (1954) aduce que la única forma a través de la cual se puede entender quién se es, es observando lo que se tiene o posee, si bien es necesario tener en cuenta que solo las posesiones hacia las que se tenga un apego emocional, más que simplemente utilitario, serán las que formarán parte del "yo extendido" (Belk, 1992). Prelinger (1959) acota, además, que las posesiones que forman parte del "yo extendido" son aquellas que se definen solo haciendo referencia a su propietario. En cuanto a las funciones que se le otorgan al "yo extendido", Belk (1988) considera que los objetos pueden ser utilizados como una herramienta o arma que permite al propietario hacer cosas que de otra forma sería incapaz, o como un objeto que permite que el propietario se vea representado de una forma simbólica.

El trabajo pionero llevado a cabo por Csikszentmihalyi y Rochberg-Halton (1981), The Meaning of Things, detalla los pormenores de una investigación llevada a cabo con 82 familias. Estos autores realizaron entrevistas con varios miembros de cada familia, en las que solicitaron una descripción exhaustiva de su vivienda y de los objetos que la contenían y eran importantes para ellos. De estas entrevistas, los autores organizaron estos objetos en 10 categorías principales, y encontraron diferencias significativas en las pertenencias favoritas con respecto a los diferentes rangos de edad (hijos, padres y abuelos) y al género de los participantes. Además, definieron por primera vez, en la literatura psicoambiental, dos dimensiones de organización de los objetos. Una primera, determinada por los polos de accióncontemplación, y una segunda cuyos polos eran orientados hacia el self-otros.

De forma análoga a esta última investigación, Kamptner (1991) y Yuste y Gonzalez (1998) llevaron a cabo dos estudios con muestras de diferentes generaciones, en los que preguntaron a los participantes por sus objetos más preciados. A continuación clasificaron estos objetos de acuerdo a las categorías elaboradas por Csikszentmihalyi y Rochberg-Halton (1981), donde obtuvieron resultados muy similares en cuanto a la preferencia de objetos en función del género y la edad de los participantes.

Otra de la investigaciones referenciales en el ámbito de los objetos y su categorización es la llevada a cabo por Prentice (1987). Esta autora trató de establecer los tipos de significados atribuidos a los objetos. Para ello realizó varias entrevistas en las que los participantes debían indicar sus cinco objetos favoritos y el porqué, lo que dio lugar a una lista de 70 objetos que fue clasificada por otros participantes en función de por qué ellos creían que podrían ser valorados. A través de estas clasificaciones, Prentice estableció dos categorías: simbólico e instrumental. La primera de ellas comprende objetos que rememoran relaciones, situaciones y sentimientos que son importantes para el individuo, mientras que la categoría instrumental engloba objetos que promueven la actividad, proporcionando beneficios directos, ya sean de disfrute o utilitarios.

En 1991, Dittmar desarrolló una investigación centrándose principalmente en encontrar diferencias de género y estatus en función de los objetos preferidos. Para ello solicitó a los participantes que cumplimentasen un cuestionario acerca de sus cinco objetos preferidos y las razones por las cuales habían sido escogidos. Al igual que los trabajos precedentes, esta autora observó dos grandes categorías. Para ella, los objetos simbólicos se vinculan a la expresión del self y a la categorización del entorno y tienen un componente emocional y cognitivo, mientras que los instrumentales son aquellos que facilitan el control sobre el entorno y tienen un componente de carácter conductual.

Atendiendo al tipo de objetos que se utilizan a la hora de personalizar los dormitorios, Aragonés y Pérez-López (2009) solicitaron a los participantes que describieran su dormitorio y los sentimientos que experimentaban en él. Con todas las descripciones se realizó un análisis de contenido, estableciendo varias categorías y subcategorías. De las categorías mencionadas, merece especial atención la denominada 
equipamiento, formada a su vez por tres subcategorías - funcional, aparatos y personal - referidas a los objetos que componen los dormitorios. Además, esta última subcategoría, personal, fue la más mencionada por todos los participantes. Estos datos parecen indicar que, considerando el dormitorio globalmente, uno de los atributos a los que comúnmente se recurre para describirlos son los objetos que contienen, y dentro de estos, aquellos que son personales y evocan experiencias pasadas del propietario.

En este trabajo se pretenden conocer los objetos que personalizan los dormitorios, y si estos se organizan en torno a las categorías de funcional y simbólico establecidas por otros autores, así como estudiar en qué medida estos objetos producen discursos diferenciados consistentes con las categorías a las que pertenecen.

Para ello, en primer lugar se obtuvo una lista de los objetos favoritos más representativos de dormitorios de estudiantes universitarios (Estudio 1). Basándose en esta lista, una nueva muestra de participantes organizó los objetos representativos según sus propios criterios (Estudio 2). Por último, se evaluaron los discursos desarrollados por los propietarios, al hablar de sus objetos favoritos (Estudio 3).

\section{Estudio 1: Selección de los objetos representativos}

\section{Método}

\section{Participantes}

Este estudio se llevó a cabo con una muestra de 64 estudiantes de la Facultad de Psicología de la Universidad Complutense de Madrid, que disponían de su dormitorio de forma exclusiva, con una edad media de 21.73 (DT = 2.38), de los cuales el $57.8 \%$ eran mujeres. Todos los participantes colaboraron de forma voluntaria.
Cuestionario y procedimiento

Los participantes completaron un cuestionario online, en el que se les preguntaba en qué medida consideraban que su dormitorio les representaba — valorado en una escala de 1 a 7 , donde 1 era "no me representa en absoluto" y 7 "me representa por completo"- y cuáles eran los 5 objetos preferidos en su dormitorio y por qué los habían escogido.

\section{Resultados y discusión}

Los resultados mostraron que los dormitorios de los participantes representaban en gran medida a sus propietarios, ya que se obtuvo una puntuación media de 5.27 (DT $=1.41)$. Los 320 objetos obtenidos inicialmente se agruparon siguiendo un consenso interjueces. En primer lugar fueron agrupados los objetos que solo se diferenciaban en el artículo, en número o eran sinónimos (como PC y ordenador). Así mismo, aquellos objetos que habían sido mencionados en una sola ocasión fueron eliminados, cuando estos podían estar representados en otros objetos (e.g. "adorno que cuelga del techo" fue incluido en "atrapasueños").

En la Tabla 1 pueden observarse los objetos resultantes que fueron asignados por los investigadores a las categorías de funcional y simbólico, de los cuales 18 se correspondían con la primera y los 29 restantes a la segunda. 


\section{Tabla 1}

Lista de objetos, clasificados según el criterio de los investigadores

\begin{tabular}{llll}
\hline \multicolumn{1}{c}{ Funcional } & \multicolumn{2}{c}{ Simbólico } \\
\hline Cama & $\begin{array}{l}\text { Despertador } \\
\text { Apuntes de } \\
\text { Ordenador }\end{array}$ & Claqueta de cine & Peluche \\
$\begin{array}{l}\text { Estantería } \\
\text { Televisión }\end{array}$ & $\begin{array}{l}\text { Coche de juguete } \\
\text { Poster }\end{array}$ & $\begin{array}{l}\text { Foto } \\
\text { Crucifijo }\end{array}$ \\
Armario & Corcho de pared & Jarrón \\
Mesa de escritorio & Estrella de mar & Diana \\
Equipo de música & Pluma de escribir & Planta \\
Cojín & Tablero ajedrez & Máscara \\
Silla & Discos y CD & Cuadro \\
Edredón & Trofeos & Figura \\
Espejo & Piedras minerales & Libros \\
Aire acondicionado & Gorro de paja & Guitarra \\
Cómoda & Bufanda de futbol & Abrecartas \\
Cortinas & Matrícula de coche & Atrapasueños \\
& Muñeca & Recuerdo de viaje \\
\hline
\end{tabular}

Teniendo en cuenta estos resultados, los siguientes estudios utilizarán los 47 objetos más representativos de los dormitorios de estudiantes universitarios. Estos objetos han resultado ser bastante similares a los obtenidos en anteriores investigaciones acerca de la categorización de objetos (Csikszentmihalyi \& Rochberg-Halton, 1981; Dittmar, 1991; Prentice, 1987), lo que permitirá la comparación de los resultados con investigaciones previas (Estudio 2). Así mismo, con los discursos referidos a estos objetos y las categorías a las que sean asignados, se comprobará si existe una relación entre el discurso que se mantiene y la categoría a la que se haya asignado cada objeto (Estudio 3).

\section{Estudio 2: Categorización de los objetos}

\section{Método}

\section{Participantes}

Los participantes de este estudio fueron 81 estudiantes de Psicología de la misma facultad, con una edad media de $22.3(D T=3.89)$, de los cuales el $51.9 \%$ eran mujeres.

\section{Cuestionario y procedimiento}

Con la lista de objetos del estudio anterior, se crearon 47 tarjetas de $10 \mathrm{~cm} \quad$ x 6 $\mathrm{cm}$, con el nombre de cada uno de los objetos. Los participantes debían organizar dichas tarjetas en tantos montones como considerasen necesarios, de tal forma que cada montón estuviese compuesto por aquellos elementos que pertenecen a una misma categoría y que no puedan ser clasificados en las categorías de los otros montones. Una vez completada la clasificación, los participantes debían indicar cada categoría creada y los objetos que la componían.

\section{Resultados}

Para conocer cuáles son las categorías subyacentes en las que se organizan los objetos de los dormitorios de los jóvenes, se construyó una matriz simétrica de similitudes de 47 x 47 . Con esta matriz se llevó a cabo un análisis de cluster jerárquico mediante el método de vinculación intergrupos, de donde se obtuvo el dendrograma que aparece en la Figura 1. 


\section{Figura 1}

Representación gráfica de las agrupaciones de los objetos obtenidas por el análisis de cluster

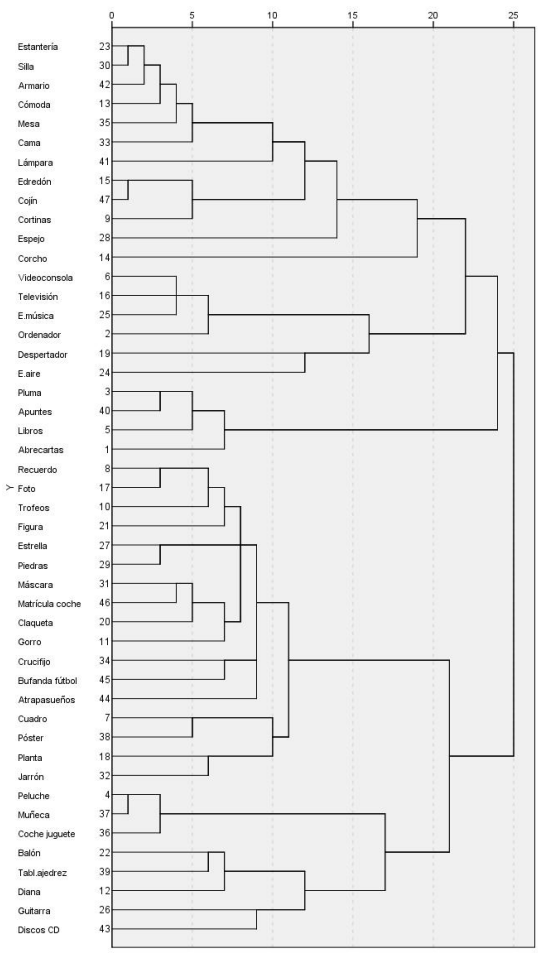

Nota. F = Funcional, $\mathrm{S}=$ Simbólico.

En el dendrograma los objetos son los nodos terminales (extremos) y la distancia entre los objetos se observa a partir de la longitud de la rama horizontal. Al mismo tiempo, cada segmento horizontal se corresponde tanto con la medida de las características comunes de los objetos que engloba, como con las características de cada uno de los objetos individualmente. Esta figura muestra una clara distinción jerárquica entre los nodos, en donde se obtiene una máxima similitud de 71, y una mínima, en la unión de todos los objetos, de 9.38. Según se puede observar en la Figura 1, aparecen dos agrupaciones claramente definidas (con similaridades entre 20.58 y 11.86), las cuales han sido etiquetadas como funcional y simbólico. A su vez, estas dos categorías se subdividen en otras dos subcategorías, etiquetadas como "equipamiento" y "profesional" — para la categoría Funcional—y "autorreferencia" y "entretenimiento" — para la categoría Simbólico-.

La categoría "funcional" engloba objetos que son prácticos y útiles para los propietarios, y que pueden ser vistos como instrumentos para alcanzar un fin o para poder manipular el entorno. La primera de sus subcategorías, denominada como "equipamiento" se caracteriza principalmente por incluir objetos de uso cotidiano, y sin los cuales no sería posible realizar muchas de las acciones y conductas que se llevan a cabo en los dormitorios. Esta subcategoría se encuentra a su vez subdividida en "mobiliario" y "aparatos electrónicos", en donde la primera se compone de objetos básicos y de uso diario, presentes en la mayoría de los dormitorios, mientras que la subcategoría de "aparatos electrónicos" está conformada por objetos de carácter electrónico o tecnológico. La segunda subcategoría, "profesional", está formada por objetos que se orientan hacia las metas u objetivos que el individuo tiene en esos momentos, en nuestro caso estudiantes, por lo que se refieren al estudio y/o enriquecimiento personal.

La categoría "simbólico" se refiere a objetos que evocan relaciones y sentimientos de los participantes, así como objetos que hacen referencia a la expresión del self. La primera de sus subcategorías, "autorreferencia", engloba objetos de decoración del dormitorio y que evocan experiencias pasadas de los propietarios. La segunda de las subcategorías, "entretenimiento", se compone de objetos que permiten al propietario disfrutar de su tiempo libre. Esta subcategoría se subdivide a su vez en "juguetes" y "hobbies", la primera de la cuales se compone de objetos que fueron utilizados durante la infancia de los propietarios y actualmente pueden considerarse recuerdos de la misma, mientras que la segunda se refiere a objetos con una alta utilidad pero que son usados con motivos de ocio y el desarrollo de habilidades principalmente recreativas. 


\section{Discusión}

El análisis jerárquico de clusters confirma que la categorización de los objetos en funcionales y simbólicos se produce a priori en la mente de las personas, lo que confirma las categorizaciones realizadas a posteriori en los trabajos de Csikszentmihalyi y Rochberg-Halton (1981), Dittmar (1991), y Kamptner (1991). Además, para cada una de estas categorías se han encontrado dos subcategorías subyacentes.

Así, dentro de la categoría funcional surgen las subcategorías "equipamiento" y "profesional". La primera de estas se subdivide en "mobiliario", que ya fue denominada por Csikszentmihalyi y Rochberg-Halton (1981) como la más mencionada por los participantes a la hora de definir las pertenencias, y comprende objetos básicos y de uso diario que se encuentran en la mayoría de los dormitorios; y "aparatos electrónicos" que se corresponde con la creada por Aragonés y Pérez-López (2009), en la que se mencionan objetos de carácter electrónico y/o tecnológico. Por último, se encuentra la subcategoría "profesional", similar en algunos aspectos a "objetos como elementos del futuro", observada por Yuste y Gonzalez (2008). Esta subcategoría está compuesta por objetos que se orientan hacia las metas $u$ objetivos de determinadas tareas productivas y/o de la ocupación que el individuo tiene. Por tanto, la categoría funcional parece abarcar objetos que tienen una función de uso y que pueden ser vistos como instrumentos para alcanzar un fin o para poder manipular el entorno.

En referencia a la categoría simbólico, los resultados han mostrado otras dos subcategorías subyacentes. La primera, denominada "autorreferencia", que contiene diferentes objetos que se utilizan para personalizar y que son recordatorios de eventos y personas que han influenciado las vidas de los participantes (Prentice, 1987). La subcategoría "entretenimiento" se encuentra en línea con la categoría "recreativa", denominada por Prentice, y se compone de "juguetes", caracterizada por abarcar objetos de la infancia sin un valor utilitario, aunque sí un valor sentimental y de rememoración de la niñez; y hobbies, definida por objetos prácticos pero que son utilizados con motivos de ocio. Así, la categoría Simbólico tiene una función de expresión del self, que rememora relaciones, situaciones y sentimientos que son importantes para el individuo (Prentice, 1987; Dittmar, 1991).

\section{Estudio 3: Discursos asociados a los objetos}

Método

\section{Participantes}

Los participantes en este estudio fueron los 64 estudiantes que participaron en el Estudio 1, quienes identificaron sus 5 objetos favoritos.

\section{Resultados y discusión}

En primer lugar, los 320 objetos obtenidos inicialmente en el estudio piloto fueron clasificados por consenso interjueces en las categorías y subcategorías obtenidas en el análisis jerárquico de cluster.

Las descripciones de los participantes acerca de por qué habían seleccionado estos objetos se sometieron a un análisis de contenido. Previamente fueron descompuestas en palabras, de tal forma que se obtuvieron 3010 palabras para funcional y 3239 para simbólico. A continuación se seleccionaron los adjetivos calificativos, que expresan los sentimientos asociados a los objetos; y los verbos, que expresan las acciones asociadas con los objetos (Aragonés, Amérigo, \& Vercher, 1997). Posteriormente, se agruparon los términos que se diferenciaban en género o número en el caso de los adjetivos y se agruparon las conjugaciones con el infinitivo, en donde se obtuvo un total de 8 adjetivos y 29 verbos diferentes.

Con el fin de conocer hasta qué punto las categorías y subcategorías se encuentran asociadas con determinados verbos y adjetivos, se 
llevaron a cabo dos análisis de correspondencia simple, cuya matriz de datos fue previamente ponderada según el número de objetos atribuidos a cada subcategoría. El primero de estos análisis, referido a los verbos, mostró que los dos ejes explican un 81.88 \% de la varianza total (Figura 2).

\section{Figura 2}

Análisis de correspondencia simple entre verbos y categorías

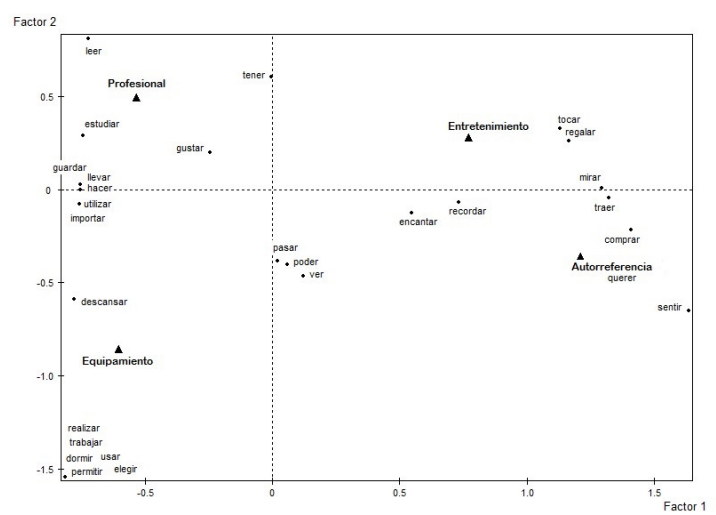

Tal y como muestra el gráfico resultante, y tomando el eje de abcisas como referencia para las subcategorías, se puede observar que los verbos están ubicados principalmente en cada uno de los extremos de este eje. Así, en el lateral izquierdo se encuentran verbos asociados con funcional, como por ejemplo estudiar, leer, hacer, usar, trabajar, entre otros; mientras que en el extremo derecho se encuentran los verbos asociados a simbólico tales como recordar, encantar, regalar, sentir, representar.

En el análisis de correspondencia simple entre adjetivos y subcategorías los dos ejes explican un $97.55 \%$ de la varianza total (Figura 3). Si se toma el eje de abcisas como referencia, se puede observar cómo los adjetivos están ubicados en cada uno de los extremos de este eje, fuertemente asociados con subcategorías funcionales y simbólicas. Asociándose a la categoría funcional, adjetivos tales como espacioso/a, imprescindible o cómodo/ a, mientras que adjetivos tales como importante, sentimental o especial se encuentran asociados con simbólico.
Figura 3

Análisis de correspondencia simple entre adjetivos y categorías

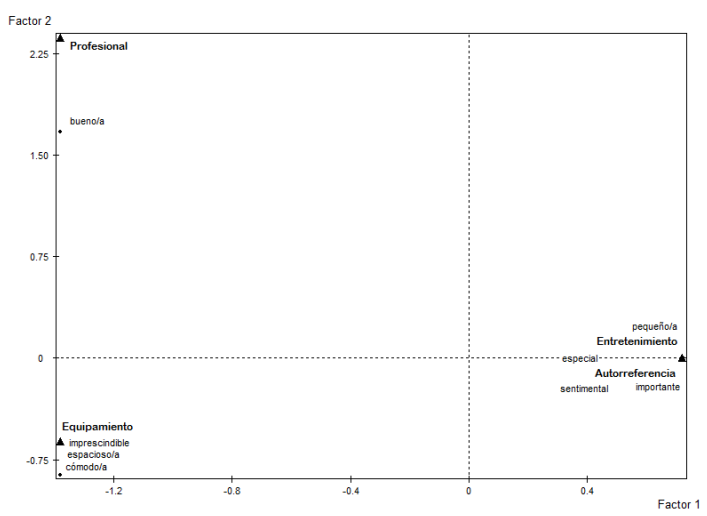

Tal y como se ha podido observar, cuando los participantes describen los objetos clasificados como funcionales, utilizan verbos con fines instrumentales, tales como estudiar, utilizar, realizar, trabajar, así como adjetivos relativos a la utilidad del objeto —esencial, cómodo, espacioso, bueno- - Sin embargo, cuando los participantes se refieren a objetos simbólicos, los verbos y adjetivos se utilizan principalmente para transmitir emociones que despiertan estos objetos - recordar, representar, amar, sentir; sentimental, especial, importante y pequeño - Estos resultados permiten confirmar que se desarrollan dos discursos distintos según la categoría a la que el objeto pertenece y que los propietarios de estos objetos, ajenos a su posterior categorización, ya realizaron esta diferencia en su discurso.

\section{Discusión general}

Los resultados obtenidos ratifican los objetivos propuestos en relación con la clasificación de los objetos en las categorías de funcional y simbólico, así como la existencia de discursos diferenciados para los objetos en función de si pertenece a una u otra categoría. Los datos obtenidos en el estudio 1 han mostrado que los participantes consideraban sus dormitorios como muy representativos de sî mismos, lo que confirma que la lista de objetos es representativa. 
Los resultados del estudio 1 han permitido contrastar con las investigaciones previas (Csikszentmihalyi \& Rochberg-Halton, 1981; Dittmar, 1991; Kamptner, 1991), que los objetos que conforman la personalización y decoración de los dormitorios de jóvenes universitarios son clasificados a priori por personas ajenas a dichos objetos en funcional y simbólico. Así mismo, el análisis de cluster ha permitido observar que para cada una de estas categorías subyacen dos subcategorías. Así, la categoría funcional se compone de las subcategorías "equipamiento" y "profesional", mientras que la categoría simbólico se compone de las subcategorías "autorreferencia" y "entretenimiento", si bien estas subcategorías podrían variar dependiendo del tipo de espacio primario estudiado y de las características sociodemográficas del ocupante.

El análisis de correspondencia llevado a cabo en el Estudio 3 pone en evidencia que existe una relación entre las categorías a las que pertenecen los objetos y las razones por las que estos objetos fueron escogidos. Si nos atenemos al significado semántico de las palabras asociadas con cada categoría, puede observarse una correspondencia entre la etiqueta de la categoría y las palabras asociadas a la misma, de tal forma que se puede inferir que las categorías de funcional y simbólico están implícitas en el discurso de los participantes.

Por otro lado, es necesario poner atención a algunas cuestiones metodológicas y posibles limitaciones de esta investigación. Atendiendo a uno de los objetivos desarrollados por Prentice (1987), podría ser necesario comprobar si el discurso que mantienen los participantes acerca de sus objetos se encuentra moderado por las creencias funcionales o simbólicas del participante, lo que podría clarificar todavía más los resultados obtenidos. Así mismo, es necesario destacar la importancia de la terminología utilizada en los cuestionarios de recogida de datos. Así, en este trabajo empírico se ha tomado la terminología y el procedimiento utilizado por Prentice, quien solicitaba a los participantes que seleccionasen sus 5 objetos "favoritos". Lo que se desea en este momento es reflexionar acerca de la idoneidad o no del término, teniendo en cuenta que, tal y como Kleine y Baker (2004) ponían de manifiesto, la utilización de diferentes adjetivos puede llevar a que los participantes seleccionen objetos diferentes. Esta limitación debería dar lugar a una investigación lingüística centrada únicamente en la variación de las respuestas obtenidas, según qué términos se utilicen, para así poder llegar a un consenso de cuál o cuáles términos son los más adecuados para obtener la esencia de los objetos que conforman el yo extendido.

\section{Agradecimientos}

Esta investigación ha sido llevada a cabo dentro del Proyecto "Percepción interpersonal a través de la personalización de la vivienda. Una aproximación desde la teoría de los cinco grandes y del modelo de contenido de los estereotipos" (PSI2010 - 17397), subvencionado por el Ministerio de Ciencia e Innovación, España.

\section{Referencias}

Aragonés, J. I. Amérigo, M., \& Vercher, M. (1997). Evaluación de un programa de educación ambiental realizado en el contexto escolar. Revista de Psicología Social Aplicada, 7, 129-143.

Aragonés, J. I., \& Pérez-López, R. (2009). Personalización del dormitorio: descripción, sentimientos y conductas. Medio Ambiente y Comportamiento Humano, $10(3), 287-301$.

Aragonés, J. I., Amérigo, M., \& Pérez-López, R. (2010). Perception of personal identity. Psicothema, 22 (4), 872-879.

Becker, F. D. (1977). Housing messages. Stroudsburg: Dowden, Hutchinson \& Ross.

Belk, R. W. (1988). Possessions and the extended self. Journal of Consumer Research, 15(2), 139-168.

Belk, R. W. (1992). Attachment to possessions. En I. Altman, \& S. M. Low (Eds.), Place Attachment (pp. 37-55). Nueva York: Plenum Press. 
Cooper, C. (1995). House as mirror of self: Exploring the deeper meaning of home. Berkeley: Conari Press.

Csikszentmihalyi, M., \& Rochberg-Halton, E. (1981). The meaning of things. Domestic symbols and the self. Nueva York: Cambridge University Press.

Dittmar, H. (1991). Meanings of Material Possessions as Reflections of Identity: Gender and Social-Material Position in Society. Journal of Social Behavior and Personality, 6(6), 165-186.

Dittmar, H. (1992). The social psychology of material possessions. To have is to be. Nueva York: Harvester Wheatsheaf.

Gosling, S. D., Craik, K. H., Martin, N. R., \& Pryor, M. R. (2005). Material Attributes of Personal Living Spaces. Home Cultures, 2(1), 51-88.

Gosling, S. D., Ko, S. J., Mannarelli, T., \& Morris, M. E. (2002). A room with a cue: Personality judgments based on offices and bedrooms. Journal of Personality and Social Psychology, 82 (3), 379-398.

Hall, J. A., \& Andrzejeski, S. A. (2008). Who draws Accurate First Impressions? Personal Correlates of Sensivity to Nonverbal Cues. En N. Ambady, \& J. J. Skowronski (Eds.), First impressions (pp. 87-105). Nueva York: Guildford.

James, W. (1890). Principios de psicología ( $1^{\text {era }}$ ed.). México D.F.: Fondo de cultura económica.

Kamptner, N. L. (1991). Personal Possessions and Their Meanings\#: A Life-Span Perspective. Journal of Social Behavior and Personality, 6(6), 209-228.

Kleine, S. S., \& Baker, S. M. (2004). An Integrative Review of Material Possession Attachment. Academy of Marketing Science Review, 1, 1-29.

Norman, D. A. (2004). Emotional design: Why we love (or hate) everyday things. Nueva York: Basic Books.

Páramo, P. (2008). La construcción psicosocial de la identidad y del self. Revista Latinoamericana de Psicología, 40(3), 539-550.
Prelinger, E. (1959). Extension and Structure of the Self. The Journal of Psychology, 48(1), 13-23.

Prentice, D. A. (1987). Psychological correspondence of possessions, attitudes, and values. Journal of Personality and Social Psychology, 53(6), 993-1003.

Sartre, J. P. (1954). El ser y la nada. Buenos Aires: Iberoamericana.

Solomon, M. R. (2008). Comportamiento del consumidor (7a ed.). México D.F.: Pearson educación.

Yuste, N., \& Gonzalez, I. (1998). Los objetos personales favoritos en la adultez y senectud. Una aproximación empírica. Anales de Psicología, 14(2), 177-192.

\section{Notas}

* Artículo de investigación. 